\title{
IMPACTOS DE EL NIÑO Y LA NIÑA EN LAS LLUVIAS DEL PERÚ (1965-2007)
}

\author{
WALDO LAVADO-CASIMIRO ${ }^{1}$, JHAN CARLO ESPINOZA ${ }^{2}$ \\ ${ }^{1}$ Servicio Nacional de Meteorología e Hidrología (SENAMHI) y Universidad Nacional Agraria La Molina, \\ Lima, Perú \\ ${ }^{2}$ Instituto Geofisico del Peru y Universidad Nacional Agraria La Molina, Lima, Peru \\ wlavado@senamhi.gob.pe,jhan-carlo.espinoza@igp.gob.pe \\ Recibido Enero de 2012 - Acepto Septiembre de 2013
}

\begin{abstract}
RESUMEN
Los impactos de El Niño (EN) y La Niña (LN) en las lluvias del Perú son evaluados utilizando datos de lluvias mensuales (1965-2007) de 155 estaciones distribuidos sobre las tres vertientes hidrográficas del Perú: 85 en la del Pacífico (VP), 21 en la del Lago Titicaca (VT) y 49 en la del Amazonas (VA). En una primera etapa, clasificamos los eventos El Niño y La Niña utilizando el Índice Troup de Oscilación del Sur (IOS) sobre la base de años hidrológicos (septiembre a agosto). Con esta información, los años se clasificaron en El Niño fuerte (ENF), El Niño moderado (ENM), La Niña moderada (LNM) y La Niña fuerte (LNF).

Los resultados muestran que sólo durante los eventos ENF y LNF se observa un alto porcentaje de estaciones con anomalías significativas de precipitación y sobre todo localizadas en la VPy VT durante el periodo de diciembre a mayo. Nuestro análisis confirma que la parte norte de la VP presenta un aumento de las lluvias durante ENF, mientras que la región sur andina de la VP presenta disminución (aumento) de lluvias durante ENF (LNF). La VT, por su parte, presenta un importante déficit de lluvias durante ENF. La variación de la precipitación en la VA en cambio, es más sensible a los eventos de LNF, durante los cuales se observan lluvias más importantes de lo normal.

En una segunda etapa, se analizan los principales modos de variabilidad interanual de las lluvias en el Perú utilizando la técnica de funciones ortogonales empíricas (EOF). Los resultados son relacionados con la variabilidad de la temperatura superficial del mar y los índices IOS, E y C del Pacífico ecuatorial. Se encontró que el principal modo de variabilidad de las lluvias (CP1, 37\% de la varianza total) está asociado a los dos eventos EN extraordinarios (1983 y 1998), lo cual generó abundantes lluvias en el norte de la VP y sequías en la VT y la VA. Por su parte, el segundo modo de variabilidad de las lluvias (CP2, 25\%) se correlaciona con las anomalías de la temperatura superficial del mar en el Pacífico ecuatorial central, condiciones frías en esta región (LN) causan más lluvias de lo normal en la región Andina de la VP, la VT y en la VA. En conclusión, se observa que la variabilidad del océano Pacífico no permite explicar la totalidad de la variabilidad pluviométrica en el Perú, por lo cual se describe como perspectiva considerar otras regiones como el Océano Atlántico Tropical.
\end{abstract}

Palabras claves: El Niño Oscilación del Sur(ENOS); Variabilidad Pluviométrica; Andes, Amazonía, Perú. ABSTRACT: IMPACTS OF EL NIÑO AND LA NIÑA IN THE PRECIPITATION OVER PERÚ
(1965-2007)
The impacts of El Niño (EN) and La Niña (LN) in rainfall in Peru are evaluated using monthly data
(1965-2007) of 155 stations distributed over the three hydrographic drainages of Peru: 85 in the
Pacific (VP), 21 in the Lake Titicaca (VT) and 49 in the Amazon (VA). To classify El Niño and La
Niña is used the Index Troup Southern Oscillation (SOI) based on hydrological years (September
to August). Using these values was reclassified as strong El Niño (ENF) moderate El Niño (ENM),
moderate La Niña (LNM) and strong La Niña (LNF).
The results show that only during ENF and LNF events exhibit a high percentage of stations with
significant anomalies of precipitation and mainly located in the VP and VT during the December to 
May period. Our analysis confirms that the northern part of the VP has an increased rainfall during ENF, while the southern Andean region of the VP has decreased (increased) rainfall during ENF (LNF). The VT presents a significant rainfall deficit during ENF. The precipitation variation in the VA is instead more sensitive to LNF events, during which major rainfall are observed than usually. In a second step, we analyze the main modes of interannual variability of rainfall in Peru using the technique of empirical orthogonal functions (EOF). The results are related to the variability of sea surface temperature and IOS, E and $\mathrm{C}$ indices of the equatorial Pacific. It was found that the main mode of rainfall variability ( $\mathrm{PC} 1,37 \%$ of the total variance) is associated with two extraordinary events EN (1983 and 1998), which generated heavy rain in the north of the VP and droughts in the VT and VA. On the other hand, the second mode of variability of rainfall (PC2, 25\%) correlates with anomalies of sea surface temperature in the central equatorial Pacific, cold conditions in this region (LN) causes more rainfall than normal in the Andean region of the VP, VT and in VA. In conclusion, it appears that the variability of the Pacific Ocean not explain the entire rainfall variability in Peru. Thus, perspectives are described considering other regions such as the Tropical Atlantic Ocean.

Keywords: El Niño Southern Oscillation (ENSO), Rainfall variability, Andes, Amazonia, Peru.

\section{INTRODUCCION}

El Niño Oscilación del Sur (ENOS) es el primer modo de variabilidad climática interanual tanto en los trópicos como a escala global (Ropelewski y Halpert, 1987). Originalmente el termino El Niño fue usado por los pescadores en Paita $\left(5^{\circ} \mathrm{S}\right)$ para describir el calentamiento del mar en el Pacífico norte peruano (desde el sur de Ecuador) y lluvias que se presentaban en época de Navidad y por períodos de varios meses (Carrillo, 1892). Bjerknes $(1966,1969)$ documenta anomalías coherentes de calentamiento del mar del Perú en la frontera con el Ecuador y relaciona este comportamiento tanto al calentamiento del mar del Pacífico tropical como a los cambios a escala planetaria de la atmosfera tropical, la "Oscilación del Sur". De ahí que se deriva el término ENOS (El Niño Oscilación del Sur). La fase cálida de ENOS es denominada El Niño (EN) mientras que la fase fría es denominada La Niña (LN; Wang et al., 2012).

Durante las últimas décadas, algunos autores han descrito los impactos de los eventos ENOS sobre las lluvias a nivel de todo el Perú, así tenemos a Tapley y Waylen (1990) quienes describen la variabilidad espacial de las lluvias anuales utilizando 18 estaciones climáticas y los eventos ENOS sobre el territorio peruano, indicando que durante los años EN el Altiplano y el sur de los Andes presentan sequías con reducciones en las lluvias anuales de hasta $18 \%$, mientras que el norte de la costa del Pacífico percibe valores superiores a $88 \%$ respecto a años no EN. Rome-Gaspaldy y Ronchail (1998) describen los comportamientos de las lluvias en Perú durante EN y LN utilizando 21 estaciones (1960-1990) encontrando que en las estaciones de la costa norte se presentan excesos significativos durante la fase EN, pero los cambios en los patrones de lluvia son diferentes de un evento a otro. Por otro lado, los autores concluyen que la pluviosidad es deficitaria en los Andes del sur durante LN. Recientemente, Lagos et al. (2008) describen la variabilidad de las lluvias relacionadas con ENOS utilizando 44 estaciones sobre el Perú, encontrando que el comportamiento de las lluvias presentan diferentes regímenes en los Andes y están moderadamente correlacionados con la temperatura superficial del mar (TSM) en la región Niño 4 (Pacífico ecuatorial central) y las lluvias en la costa norte están estrechamente relacionados con la zona Niño 1+2 (Pacífico ecuatorial Este). En general, como describe Lavado et al. (2012a) las lluvias y los caudales en el norte de la vertiente del Pacífico peruano y en la vertiente del lago Titicaca, son influenciadas de manera inversa por la variabilidad del ENOS. Así, durante El Niño, las lluvias y caudales están sobre sus valores normales en la costa norte mientras que en la vertiente del Titicaca y al este de los Andes, se presentan sequías hidrológicas (Waylen y Caviedes, 1986; Ropelewski y Halpert, 1987; Aceituno, 1988; Tapley y Waylen, 1990; Rome-Gaspaldy y Ronchail, 1998; Vuille et al., 2000; Waylen y Poveda, 2002; Romero et al., 2007, entre otros). Adicionalmente, recientes trabajos han documentado el rol de La Niña y teleconexiones asociadas en las intensas precipitaciones y elevados caudales en la cuenca amazónica peruana (Espinoza et al., 2012; Espinoza et al., 2013). Según los autores, las teleconexiones asociadas a La Niña generan un incremento del transporte de humedad proveniente del Atlántico tropical norte y del Mar Caribe hacia el oeste de la cuenca amazónica, así como un debilitamiento del Monzón sudamericana. Estos mecanismos se traducen en un incremento de la convergencia del transporte de humedad en la cuenca amazónica peruana, como ocurrió durante Noviembre 2011 y Abril de 2012, produciendo el caudal más elevado que se tenga registro en el río Amazonas.

Los eventos El Niño que tuvieron mayor impacto en la economía peruana como consecuencia de los desastres que causaron las inundaciones producto de las fuertes lluvias y las sequías, fueron los de los años 1982-1983 y 1997-1998. Así, de acuerdo a la OPS (2000) las pérdidas directas causadas por El Niño durante el evento extremo 1997-1998 se estimaron 
en US\$ 1000 millones, de los cuales 800 corresponden al Norte del Perú (inundaciones) y 200 millones a la Sierra sur (sequías). El impacto económico de este desastre se reflejó en la disminución significativa del producto bruto interno (PBI) del Perú, que descendió hasta en un $12 \%$ y que, relacionado con el incremento de la población, originó una notable caída del PBI per cápita del $14.3 \%$.

Debido a la importancia económico-sociales de las variaciones de la TSM en el Pacífico ecuatorial sobre territorio peruano, y teniendo en cuenta que los estudios de estos impactos han estado generalmente restringidos a zonas puntuales, el presente trabajo tiene como objetivo describir los impactos de la TSM en el pacífico ecuatorial en los patrones de lluvia tanto a escala anual (año hidrológico) como a escala estacional, a través de todo el territorio peruano considerando una gran red de estaciones (155) analizadas por su distribución en vertientes hidrológicas.

\section{DATOS EMPLEADOS}

\section{1 Î́ndice de Oscilación del Sur (IOS) y eventos ENOS}

En una primera etapa, utilizamos el índice de oscilación del sur (IOS) para caracterizar los eventos El Niño y La Niña. El IOS es calculado por The Australian National Climate Centre y los datos fueron obtenidos de la web de esta institución (www.bom.gov.au/climate/current/soihtm1.shtml). Este índice es bastante usado para caracterizar la variabilidad del ENOS sobre todo en el sur este de Asia y en la región del Pacífico (Xu et al., 2004). El IOS es estimado de las diferencias de valores mensuales de presión del aire entre Tahití (aproximadamente $150^{\circ} \mathrm{O}, 18^{\circ} \mathrm{S}$ ) y Darwin (aproximadamente $130^{\circ} \mathrm{E}, 13^{\circ} \mathrm{S}$ ). En la bibliografía existen diferentes métodos de estimación del IOS, pero ha sido probado que la diferencia entre estos métodos es bastante pequeña (Kawamura et al., 1998). El método utilizado en este estudio es el Troup IOS, que es el valor estandarizado de la anomalía del promedio de presión a nivel del mar (MSLP por sus siglas en ingles) de la diferencia entre Tahití y Darwin y expresado como sigue:

$$
S O I=10 X \frac{D_{M S L P}-\overline{D_{M S L P}}}{S_{M S L P}}
$$

Donde: $D_{M S L P}$ es la diferencia entre el promedio mensual MSLP en Tahiti y Darwin; $\bar{D}_{M S L P}$ es el valor medio de $D_{M S L P}$ y $\mathrm{S}_{\mathrm{MSLP}}$ es la desviación estándar de $D_{M S L P}$ sobre todo sobre el período 1965-2007.

Para propósitos de evaluar los impactos de los eventos ENOS clasificados en función del IOS, en este estudio se utilizaron los valores suavizados de 6 meses del IOS similar a lo utilizado por McKerchar et al. (1998); Chiew et al.(1998);
Cluis y Laberge (2002) y Xu et al. (2004). Así tenemos que los años La Niña se definen para valores de IOS superiores a 5; los años El Niño se definen para valores de IOS inferiores a -5 y los años normales se definen para valores de IOS entre -5 y 5 .

Para este primer análisis se clasificaron los años El Niño fuerte (ENF) para los valores del IOS menores a -10; los años El Niño moderado (ENM) entre -10 y -5 ; La Niña fuerte (LNF) para los valores mayores a 10 y los años La Niña moderada (LNM), entre 5 y 10. En total se tienen 3 años hidrológicos para los años ENF (1982-83, 1991-92 y 1997-98), 11 para los ENM (1965-66, 1972-73, 1977-78, 1986-87, 1987-88, $1992-$ 93, 1993-94, 1994-95, 2002-03, 2004-05 у 2006-07), 4 para las LNF (1970-71, 1973-74, 1975-76, 1988-89) y 4 para LNM (1974-75, 1998-99, 1999-2000 у 2000-2001).

\subsection{Variabilidad de la TSM en el Pacífico Ecuatorial}

Con la finalidad de evaluar la relación entre la variabilidad pluviométrica en el territorio peruano y la TSM en el pacífico ecuatorial, se usaron dos índices oceánicos recientemente definidos, los índices $\mathrm{C}$ y E (http://www.met. igp.gob.pe/datos/EC.txt). Estos índices resultan utiles ya que sintetizan la variabilidad del Pacífico ecuatorial central (C) y la del Pacífico ecuatorial Este (E). Adicionalmente, ambos índices son, por construcción no correlacionados entre sí (Takahashi et al., 2011). Por último, utilizamos los valores globales de la TSM obtenidos de la base de datos SST v3b (Smith et al., 2008) que se descarga libremente de: http://www.esrl.noaa.gov/psd/data/.

\subsection{Precipitación observada en el territorio peruano}

Para el presente trabajo se utilizaron 155 estaciones con información de lluvia mensual distribuidas en las tres vertientes hidrográficas del Perú: 85 sobre la vertiente del Pacífico (VP), 21 sobre la vertiente del Titicaca (VT) y 49 sobre la vertiente Amazónica (VA; ver Figura 1). Esta información proviene del SENAMHI - Perú. La información de lluvia fue analizada y completada con estaciones vecinas siempre y cuando las correlaciones tuvieran un nivel de significancia por encima del 95\%. La información comprende el periodo de Septiembre de 1965 hasta Agosto de 2007.

Las lluvias sobre las vertientes de análisis, presentan promedios multianuales diversos durante 1965-2007. En general los mayores módulos se dan en la parte baja (llano amazónico) y en bajas latitudes (norte) de la VA y las menores lluvias se observan en la VP cerca al Océano Pacífico y en bajas latitudes. Asimismo, la VT presentan mayores lluvias en bajas altitudes y en zonas localizadas al norte. Para mayores detalles sobre la repartición espacial de las lluvias en el Perú, ver Lavado et al., (2012a). 


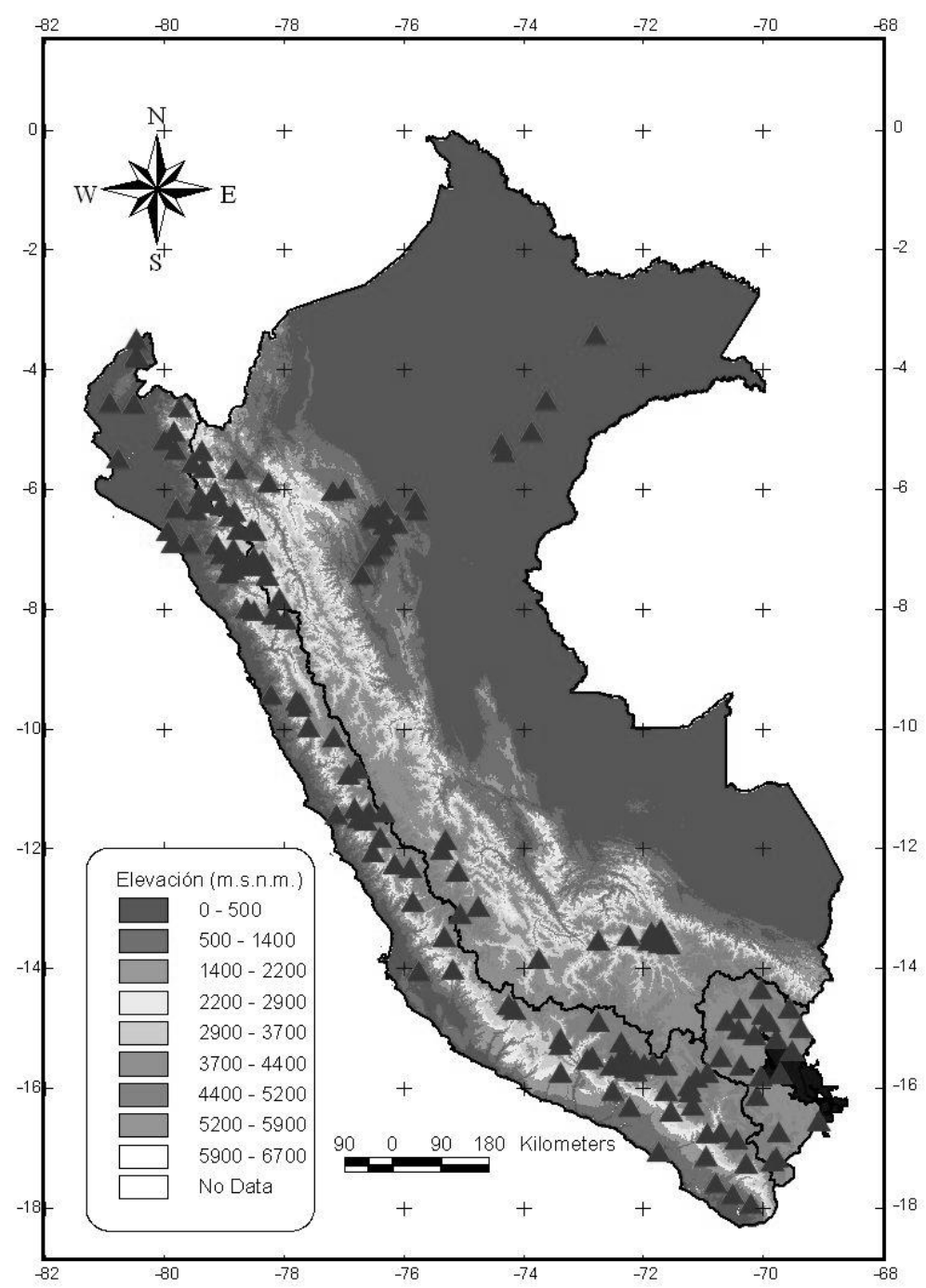

Figura 1 - Ubicación espacial de las estaciones utilizadas en este estudio, localizadas en las vertientes (líneas negras) del: Pacífico (Oeste); Lago Titicaca (Sur este) y Amazonas (Este).

\section{DESCRIPCION DE LA METODOLOGIA}

La metodología utilizada en este estudio comprende las siguientes etapas:

\section{a) Para la relación entre el ENSO y las anomalías de precipitación}

i) Identificación de la diferencia de las lluvias (Anomalías) entre los años clasificados (La Niña y El Niño) y el promedio histórico (1965-2007). Para este fin, primeramente son clasificados los años hidrológicos (Septiembre (año k-1) a Agosto (año k)) en años El Niño y años La Niña. Estas anomalías son calculadas para cada una de las estaciones pluviométricas analizadas.

ii) Cuantificación de la significancia estadística utilizando el test de Montecarlo, para lo cual se generaron 1000 muestras tomadas al azar del período histórico (1985-2007) a los cuales se les estimo las anomalías tal como se describe en el punto i). Luego, la significancia estadística (p-valor) se estimó como la proporción del número de casos comparables con las anomalías estimadas en i) (más extremas de las encontradas) entre el total de muestras (1000). Finalmente, en el presente artículo sólo se discuten aquellas estaciones que presentaron p-valores por debajo de 0.05 , es decir, con significancia estadística de $95 \%$.

iii) Técnicas estadísticas de correlación lineal (Pearson) entre las lluvias estandarizadas versus el índice Troup IOS, siempre analizando sólo aquellas estaciones donde la correlación tenga una significancia superior al $95 \%$.

Adicionalmente, se analizan los impactos estacionales (trimensuales) del ENOS sobre las lluvias en el Perú, para lo cual se dividen las series de lluvias en función a la clasificación de las estaciones en el hemisferio Sur, es decir: verano 
(Diciembre-Enero-Febrero, DEF); primavera (SeptiembreOctubre-Noviembre, SON), otoño (Marzo-Abril-Mayo, MAM) e invierno (Junio-Julio-Agosto, JJA). Sobre las series trimestrales se desarrollara los pasos metodológicos i), ii) y iii).

b) Para la identificación de los principales modos de variabilidad pluviométrica y su relación con la TSM del pacífico ecuatorial

Para este propósito se calcularon los principales modos de variabilidad interanual de las lluvias en el Perú mediante la técnica de funciones ortogonales empíricas (EOF, Dillon and Goldstein, 1984). Los resultados (componentes principales o CPs) son relacionados con la variabilidad de la TSM del Pacífico ecuatorial utilizando tanto los índices E y C, como la TSM global descritos anteriormente. Este análisis permitirá complementar la metodología propuesta en la parte a) mediante: i) reconocer cuáles son los principales modos de la variabilidad espaciotemporal de las lluvias en el Perú durante el periodo 1967-2007 y ii) identificar la relación de estos modos con la variabilidad de la TSM de las diferentes regiones del Pacífico ecuatorial, usando índices que describen de manera independiente la variabilidad del Pacífico central y del este (Takahashi et al., 2011).

\section{ANOMALIAS DE LAS LLUVIAS DURANTE LOS AÑOS EL NIÑO Y LA NIÑA}

\subsection{Precipitaciones anuales (Año hidrológico)}

La Figura 2 muestra los impactos de los eventos ENOS sobre las lluvias anuales (en valores de anomalías) para el periodo 1965-2007 y clasificados en ENF (Figura 3a), ENM (Figura 3b), LNF (Figura 3c) y LNM (Figura 3d). El porcentaje de estaciones con anomalías significativas es de 44\% (68 de 155 estaciones) para ENF, 5\% (7 de 155) para ENM, 26\% (40 de 155 ) para LNF y $8 \%$ (12 de 155) para LNM. En general, se observa que tanto LNF y LNM están asociadas a un aumento significativo de la precipitación en la VA, la VT y región andina,

\section{b) ENM Anual}
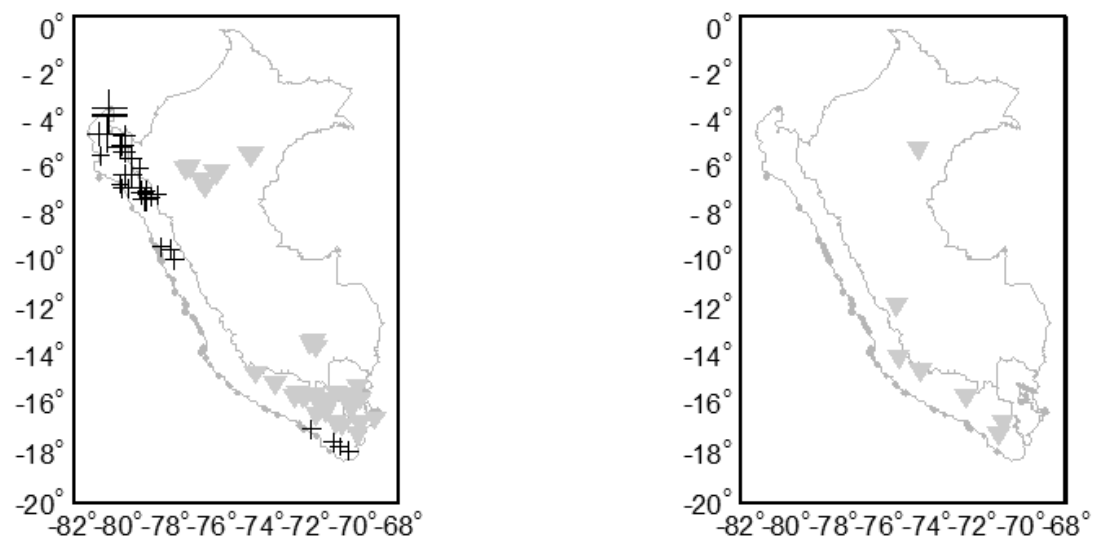

c) LNF Anual

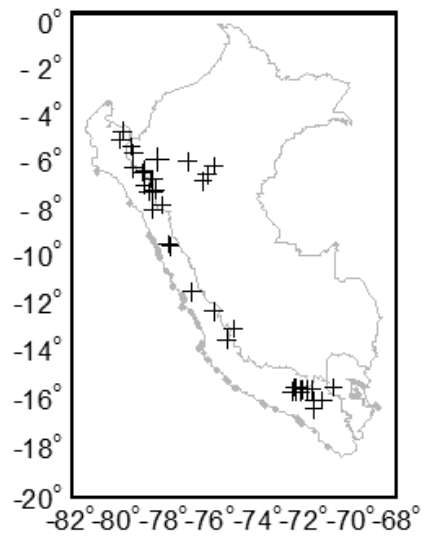

d) LNM Anual

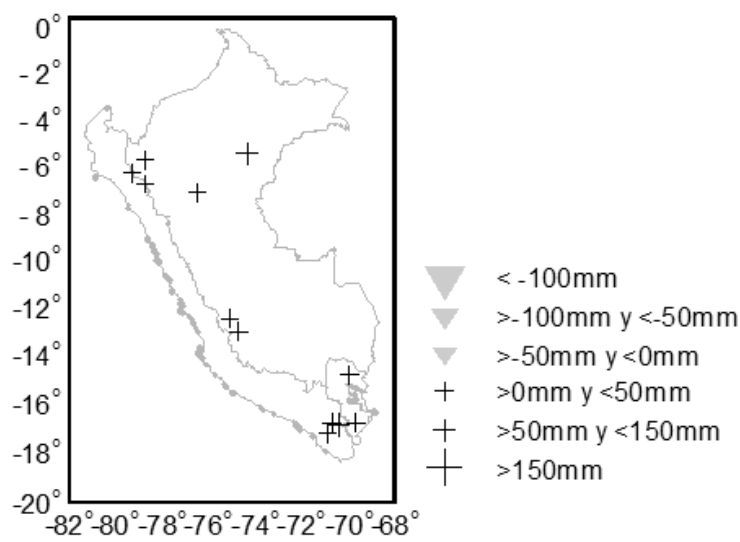

Figura 2 - Gráfica espacial de los cambios en mm de las medias de lluvias anuales respecto a su serie histórica (1965-2007), para los diferentes eventos. Anomalías de las estaciones representadas en esta figura tienen una significancia al 95\% usando el test de Montecarlo. 


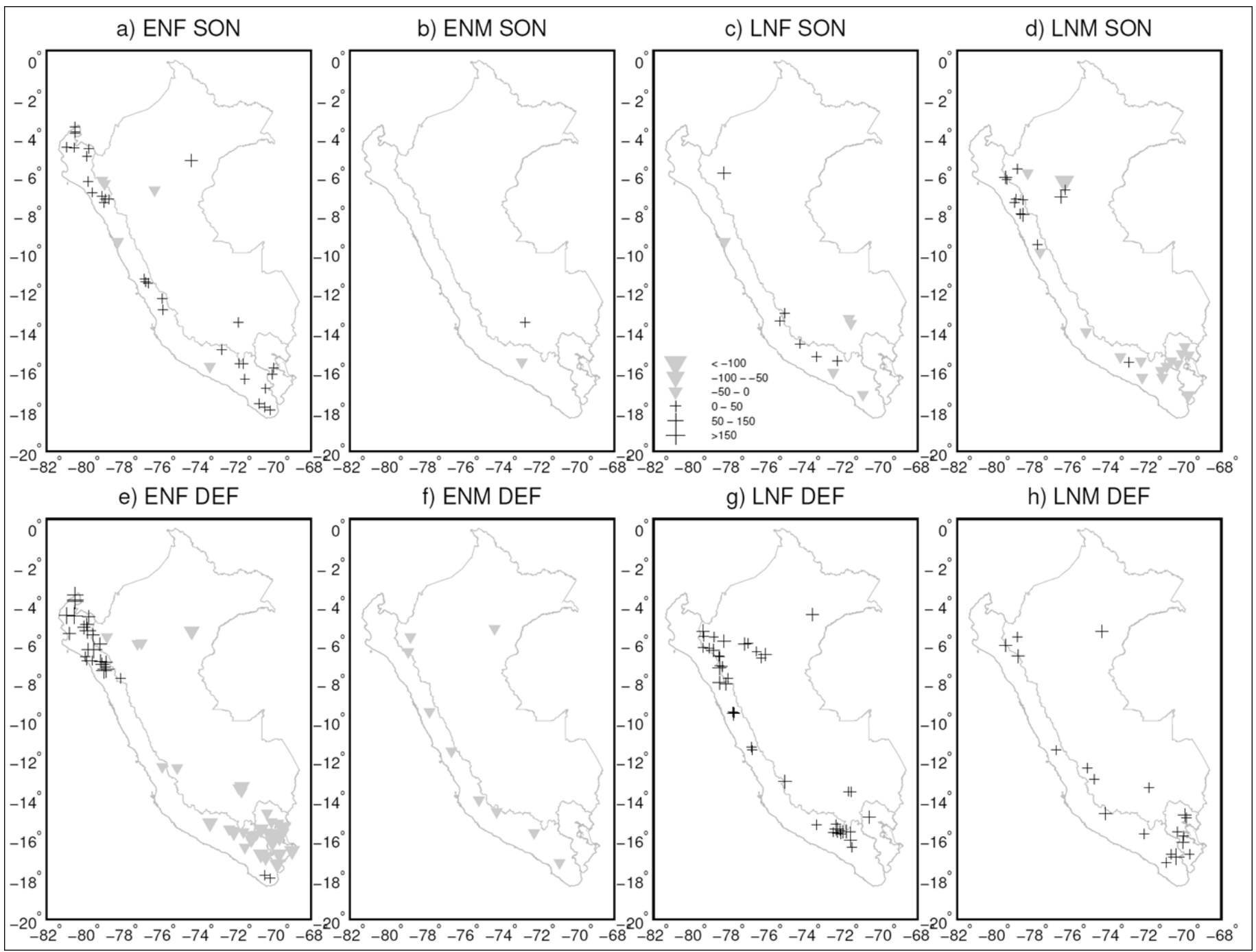

Figura 3 - Gráfica espacial de los cambios en mm de las medias de lluvias para los trimestres SON y DEF respecto a su serie histórica (1965-2007) para los diferentes eventos. Anomalías de las estaciones representadas en esta figura tienen una significancia al 95\% usando el test de Montecarlo.

mientras que durante ENM existe una disminución de las lluvias en la VA y la VT. Por su parte durante ENF existe también una disminución de las lluvias en la VA y VT, pero un exceso de lluvias en la VP, el cual es más acentuado en la costa norte.

Durante los eventos ENF (Figura 3a) se observan anomalías significativas positivas (negativas) en un número de: 29 (16) de 85 en la VP, 3 (9) de 49 en la VA y 11 de 21 con anomalías negativas en la VT. Estas anomalías han sido del orden de hasta $+220 \mathrm{~mm}$ localizado sobre todo en la zona norte de la VP pero en esta misma vertiente, en la zona sur, y por encima de los $2400 \mathrm{msnm}$ se observan anomalías negativas hasta de $-30 \mathrm{~mm}$ (ver Figura 3a) mientras en la VT están anomalías son negativas hasta de $-30 \mathrm{~mm}$. Caso aparte es la VA, donde las anomalías negativas estimadas no son homogéneas espacialmente y se presentan en sólo 12 de 49 estaciones. Los eventos ENM (Figura 3b) presentaron anomalías negativas dispersas y en un número ínfimo de estaciones (7 de 155) sobre el Perú.

Durante LNF se presentaron anomalías significativas positivas, así se tienen 24 de 85 en la VP, 1 de 21 en la VT y 15 de 49 en la VA. En la VP están localizadas entre 1000 y 4000 msnm y entre las latitudes 4 a $16^{\circ} \mathrm{S}$ y con anomalías que llegan a $+40 \mathrm{~mm}$ mientras en la VA están localizadas sobre todo en la zona noroeste con anomalías de hasta $+86 \mathrm{~mm}$. Los eventos LNM (Figura 3d) presentaron anomalías positivas dispersas y en un número menor de estaciones (12 de 155) sobre el Perú.

\subsection{Impactos en las lluvias estacionales}

La Tabla 1 muestra el número de estaciones que presentaron anomalías positivas y negativas significativas para cada uno de los trimestres seleccionados y para cada 
Tabla 1 - Número de estaciones con anomalías significativas al 95\% considerando cada uno de los eventos seleccionados y para cada uno de los trimestres. El Niño fuerte (ENF), El Niño moderado (ENM), La Niña fuerte (LNF) y La Niña moderada (LNM). T es el número total de estaciones para cada vertiente.

\begin{tabular}{|c|c|c|c|c|c|c|c|c|c|c|c|c|}
\hline & \multicolumn{12}{|c|}{ SON } \\
\hline & \multicolumn{4}{|c|}{ Pacífico (T=85) } & \multicolumn{4}{|c|}{ Titicaca $(T=21)$} & \multicolumn{4}{|c|}{ Amazonas $(\mathrm{T}=49)$} \\
\hline & ENF & ENM & LNF & LNM & ENF & ENM & LNF & LNM & ENF & ENM & LNF & LNM \\
\hline Positivos & 26 & 0 & 4 & 8 & 2 & 0 & 0 & 0 & 2 & 1 & 2 & 4 \\
\hline \multirow[t]{3}{*}{ Negativos } & 2 & 1 & 3 & 8 & 0 & 0 & 0 & 7 & 3 & 0 & 2 & 2 \\
\hline & \multicolumn{12}{|c|}{ DEF } \\
\hline & ENF & ENM & LNF & LNM & ENF & ENM & LNF & LNM & ENF & ENM & LNF & LNM \\
\hline Positivos & 23 & 0 & 22 & 7 & 0 & 0 & 1 & 6 & 3 & 0 & 20 & 6 \\
\hline \multirow[t]{3}{*}{ Negativos } & 12 & 6 & 0 & 0 & 14 & 0 & 0 & 0 & 8 & 3 & 0 & 0 \\
\hline & \multicolumn{12}{|c|}{ MAM } \\
\hline & ENF & ENM & LNF & LNM & ENF & ENM & LNF & LNM & ENF & ENM & LNF & LNM \\
\hline Positivos & 27 & 1 & 18 & 2 & 0 & 1 & 1 & 0 & 3 & 3 & 11 & 2 \\
\hline \multirow[t]{3}{*}{ Negativos } & 23 & 3 & 3 & 4 & 12 & 0 & 0 & 2 & 6 & 1 & 0 & 1 \\
\hline & \multicolumn{12}{|c|}{ JJA } \\
\hline & ENF & ENM & LNF & LNM & ENF & ENM & LNF & LNM & ENF & ENM & LNF & LNM \\
\hline Positivos & 11 & 0 & 9 & 15 & 0 & 0 & 0 & 0 & 0 & 0 & 7 & 8 \\
\hline Negativos & 12 & 13 & 7 & 2 & 0 & 11 & 0 & 0 & 8 & 10 & 0 & 0 \\
\hline
\end{tabular}

vertiente. Haciendo un resumen general, se tiene que para SON el porcentaje de estaciones con anomalías significativas fue de 23\% (35 de 155) durante ENF, 1\% (2 de 155) durante ENM, 7\% (11 de 155) durante LNF y 19\% (29 de 155) durante LNM; para DEF el porcentaje de estaciones con anomalías significativas fue de 39\% (60 de 155) durante ENF, 6\%(9 de 155) durante ENM, 28\% (43 de 155) durante LNF y 12\% (19 de 155) durante LNM; para MAM el porcentaje de estaciones con anomalías significativas fue de 46\% (71 de 155) durante ENF, 6\% (9 de 155 ) durante ENM, 21\% (33 de 155) durante LNF y 7\% (11 de 155) durante LNM; para JJA el porcentaje de estaciones con anomalías significativas fue de $20 \%$ (31 de 155) durante ENF, $22 \%$ (34 de 155) durante ENM, 15\% (23 de 155) durante LNF y $16 \%$ (25 de 155) durante LNM.

Las Figuras 3a-3d representan espacialmente las estaciones con anomalías significativas para el trimestre SON. Se observa que durante el ENF las anomalías en este trimestre están localizadas sobre todo en la VP (26 positivas y 2 negativas) y se centran a lo largo de esta vertiente con anomalías positivas que van hasta $+30 \mathrm{~mm}$. Durante ENM (Figura $3 b$ ) y LNF (Figura 3c) solo se observan anomalías aisladas. La Figura 3d muestra las anomalías para el trimestre SON durante LNM donde se puede observar que el numero de anomalías positivas y negativas está repartida en cantidad similar ( 8 ver Tabla 1 ), estando las positivas localizadas en el norte sobre $8^{\circ} \mathrm{S}$ y las negativas localizadas hacia el sur de esta referencia geográfica y los módulos de anomalías varían desde $+55 \mathrm{~mm}$ las positivas a $-18 \mathrm{~mm}$ las negativas.
Las Figuras 3e-3h muestra las anomalías para el trimestre DEF. Durante ENF (Figura 3a) se puede observar que el mayor porcentaje de estaciones con anomalías significativas negativas (14 de 21) se dan en la VT y se centran a lo largo de esta vertiente con anomalías que van hasta $-118 \mathrm{~mm}$. También, es resaltante el número de estaciones con anomalías positivas significativas en la VP (23) que están localizados sobre todo en el norte (desde $8^{\circ} \mathrm{S}$ ) de esta vertiente con módulos de hasta +380 mm pero también hay presencia de anomalías negativas hacia el sur de $12^{\circ} \mathrm{S}$ con módulos de hasta $-80 \mathrm{~mm}$. La VA exhibe algunas anomalías significativas negativas pero bastante dispersas espacialmente. La Figura $3 \mathrm{~g}$ muestra las anomalías para el trimestre DEF durante LNF, todas estas son positivas y se dan sobre todo en la región andina de la VP (26\%) y la VA (41\%). En la VP están localizados a lo largo de esta vertiente pero centradas entre 2000 y 4000 msnm. Los módulos de estas anomalía van hasta $+70 \mathrm{~mm}$ en la VPy hasta $+120 \mathrm{~mm}$ en la VA. Durante DEF, ENM (LNM) muestra anomalías significativas negativas (positivas) en algunas estaciones del territorio peruano pero llegando ha ser el número de ellos no muy significativo (menos del 10\% del total de estaciones).

La Figura 4a muestra las anomalías para el trimestre MAM durante ENF donde se puede observar que el mayor porcentaje de estaciones con anomalías significativas positivas y negativas se dan en la VP y VT (Tabla 1). En la VP las anomalías positivas de hasta $+471 \mathrm{~mm}$ se dan sobre todo al norte de $12^{\circ}$ $\mathrm{S}$ y por debajo de los $3000 \mathrm{msnm}$ pero también hay presencia de anomalías negativas hacia el sur de $12^{\circ} \mathrm{S}$ y por encima de 
a) ENF MAM

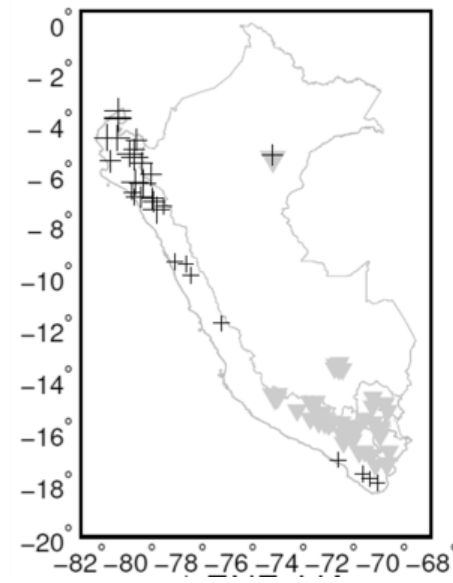

b) ENM MAM

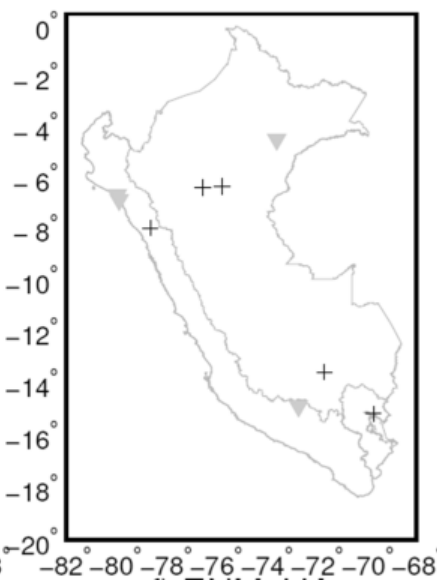

c) LNF MAM

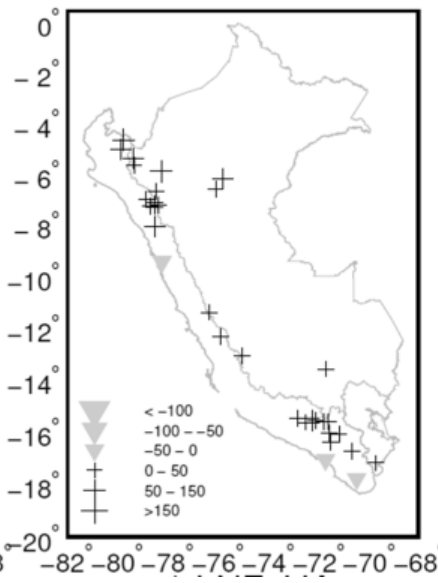

d) LNM MAM

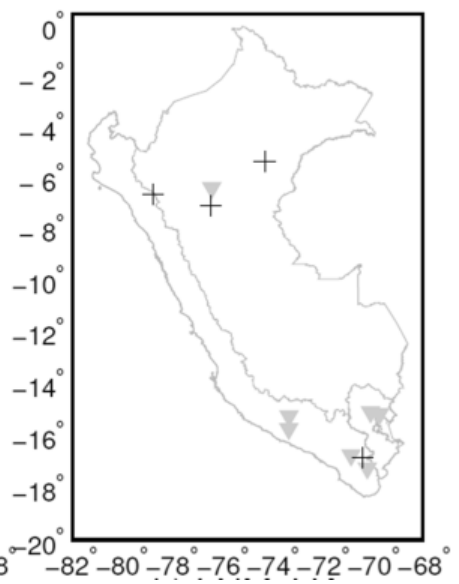

Figura 4 - Gráfica espacial de los cambios en mm de las medias de lluvias para los trimestres MAM y JJA respecto a su serie histórica (1965-2007) para los diferentes eventos. Anomalías de las estaciones representadas en esta figura tienen una significancia al 95\% usando el test de Montecarlo.

los 3000 msnm con módulos de hasta $-51 \mathrm{~mm}$. La VA exhibe algunas anomalías significativas negativas (12) con módulos de hasta -34 mm. La Figura 4c muestran las anomalías para el trimestre MAM durante LNF, todas estas son en su mayoría positivas y se dan sobre todo en la VP (25\%) y VA (22\%). En la VP están localizados a lo largo de esta vertiente pero con mayores módulos de anomalías (de hasta $+89 \mathrm{~mm}$ ) en la parte norte mientras en la VA están dispersos sobre esta vertiente pero con mayores módulos (de hasta $+124 \mathrm{~mm}$ ) en la parte norte sobre los $7^{\circ} \mathrm{S}$.

En conclusión, nuestro análisis confirma que la parte norte de la VP presenta un aumento de las lluvias durante ENF, las cuales se dan principalmente entre los meses de septiembre a mayo, mientras que la región sur andina de la VP presenta disminución de lluvias durante ENF en el periodo de diciembre a agosto. Nuestro análisis también revela que la región andina de la VP sufre un aumento de lluvias durante LNF, la cual se da principalmente entre los meses de diciembre a agosto. La VT, por su parte, presenta un importante déficit de lluvias durante ENF entre los meses de diciembre a mayo (coincidente con la época de lluvias en esta región). La variación de la precipitación en la VA en cambio, es más sensible a los eventos de LNF, durante la cual se observan lluvias más importantes de lo normal, en especial durante el periodo de diciembre a agosto (Figuras 3 y 4).

En el presente trabajo se analiza el IOS como índice para clasificar temporalmente los eventos ENOS. Sin embargo, la climatología del Océano Pacífico Tropical muestra la existencia de diversos "modos" de El Niño conocidos como el Niño del Pacifico Central (Yeh et al., 2009) y Modoki (Ashok et al., 2007) que han sido recientemente reinterpretados por Takahashi et al. (2011).
En este contexto, y para profundizar nuestros resultados, analizamos la relación existente entre las lluvias observadas y las variaciones de la TSM en la región del Pacífico central (índice C) y en la región del Pacífico Este (índice E). Los resultados muestran que existe una correlación directa y significativa al 95\%, entre el índice E y las lluvias en la costa peruana, siendo esta más extendida en la parte norte de la VP (Figura 5a). Por otro lado, la correlación es inversa y significativa al 95\% entre el índice $\mathrm{C}$ y las lluvias en la región Andina de la VP y en un número más reducido en la VT y la región andina de la VA (Figura 5b). Estos resultados confirman que valores positivos del índice E (El Niño costero) están asociados a más lluvia de lo normal en la región costera (en particular la VP norte), mientras que valores negativos del índice $\mathrm{C}$ (La Niña) se relacionan con más lluvia de lo normal en la región Andina, la VT y la VA.

\section{VARIABILIDAD PLUVIOMETRICA Y ANOMALIAS DE LA TEMPERATURA SUPERFICIAL DEL MAR}

Considerando que este trabajo se basa en un número importante e estaciones pluviométricas en el territorio peruano, a continuación se describen los principales modos de variabilidad espacio-temporal de las lluvias y luego, estos modos son relacionados con la TSM global y los índices IOS, C y E. Inicialmente se calcularon los principales modos de variabilidad de las lluvias anuales utilizando la técnica de funciones ortogonales empíricas (EOF). Así, el primer componente principal (CP-1, Figura 6a) explica el $37 \%$ de la variancia y está asociado a las variaciones de la TSM en el Pacífico Este (Figura 6b), en particular a las fuertes anomalías positivas ocurridas en los dos eventos El Niño extraordinarios (1983 y 1998) el cual produce: 
i) abundantes lluvias en la costa peruana, especialmente en la costa norte (Figura 6c). Este fenómeno ha sido tradicionalmente asociado con altas temperaturas de la TSM de la región cercana a la costa norte y un incremento de vientos del oeste de bajo nivel sobre la costa norte del Perú, lo cual favorece la convección en el flanco Oeste de los Andes (Takahashi et al., 2004). ii) En general, menos precipitación en la cuenca amazónica (Figura 6c) asociada a un debilitamiento de los vientos alisios del noreste y un incremento del LowLevel Jet (LLJ) (Marengo et al., 2004), lo cual produce una divergencia del flujo de humedad en el oeste de la cuenca amazónica, como mostrado en Espinoza et al., (2011) para el caso de 1998.
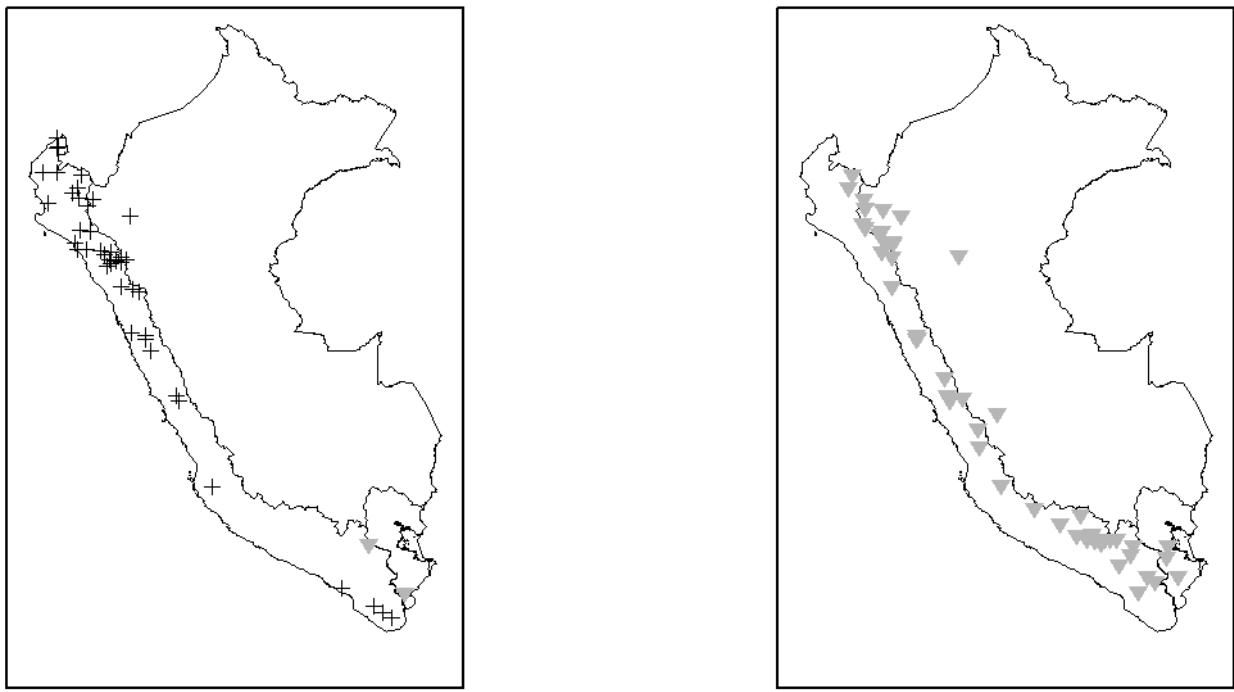

Figura 5 - Correlaciones significativas al 95\% entre as lluvias anuales y los valores medio anuales de a) índice E y b) índice C. Los triángulos grises hacia abajo representan correlaciones negativas y los signos negros "mas" representan correlaciones positivas.

CP-1 $37 \%$
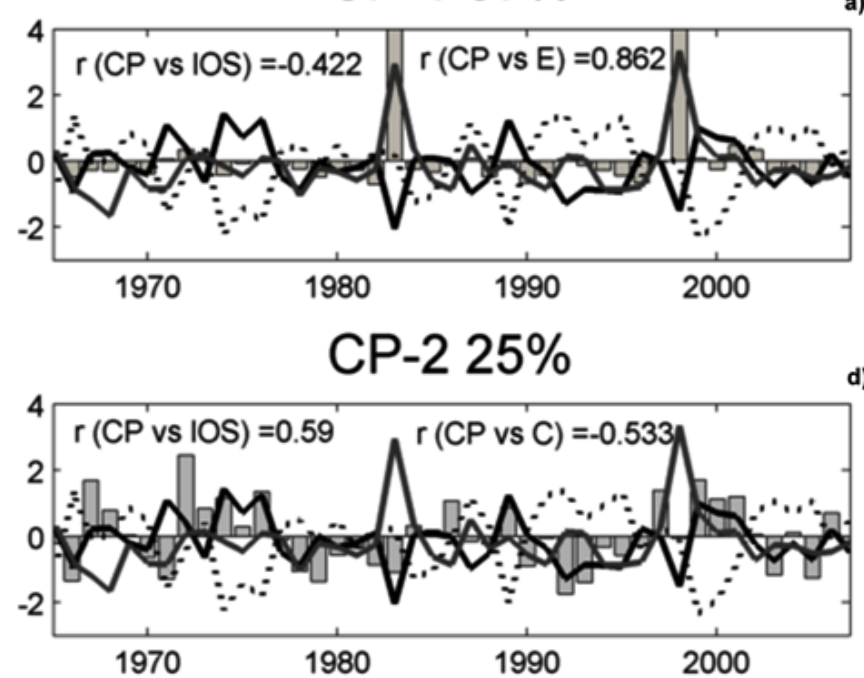

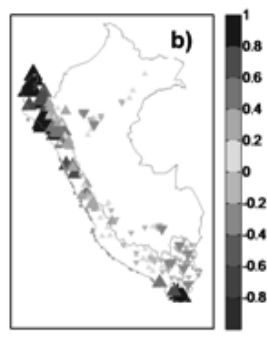

d)

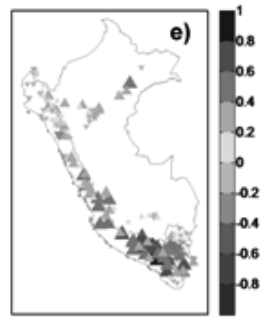

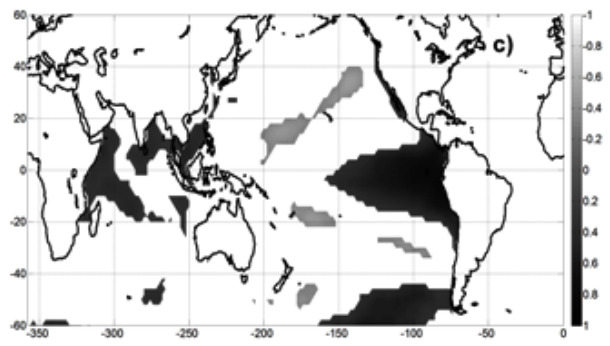

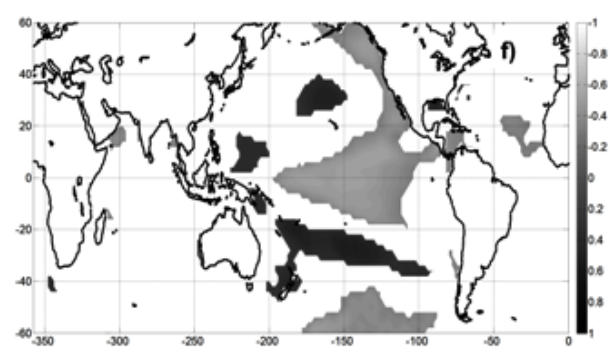

Figura 6 - Valores anuales (Septiembre a Agosto) para el período 1965-2006 de a y d) Componentes principales 1 y 2 (barras grises) versus IOS (línea negra), E (línea gris) y C (línea punteada), solo los r (coeficiente correlación) significativos son mostrados; b y e) correlaciones significativas entre los componentes principales y la Temperatura superficial del mar (TSM); c y f) "loadings" de los componentes principales en cada estación de precipitación utilizada. 
Por otro lado, el CP-2 (Figura 6d) explica el 25\% de la variabilidad interanual de las lluvias en el Perú. Los valores altos de CP-2 están asociados a condiciones más frías de lo normal en en el Pacífico ecuatorial central (eventos La Niña, Figura 6e). Estos eventos producen más lluvias de lo normal en los Andes y en la cuenca amazónica (Figura 6f). Valores altos del CP-2 corresponden a años de crecidas excepcionales del río Amazonas $(1971,1986,1989,1999)$ como documentado en Espinoza et al (2013). LN influencia las precipitaciones de la cuenca amazónica mediante procesos de teleconexión. Condiciones más frías de lo normal en la TSM del Pacifico ecuatorial genera un tren de ondas en la altura de geopotencial a $850 \mathrm{hPa}$ con anomalías positivas sobre el sur y norte del Atlántico. Estas anomalías favorecen una mayor entrada de humedad hacia el oeste de la cuenca amazónica y una retención de los vientos húmedos en el oeste Amazónico, generando una mayor convergencia de humedad sobre la Amazonía peruana (Espinoza et al., 2013).

Es importante mencionar que la correlación entre el IOS y el CP1 resulta relativamente modesta $(\mathrm{r}=-0.42)$, mientras que la variación en la TSM del Pacífico Este, sintetizada por el índice E, explican considerablemente mejor la variabilidad del CP-1 ( $\mathrm{r}=0.86)$. Para el caso del CP2, ambos índices IOS y la variación de la TSM del Pacífico central, sintetizada por el índice $\mathrm{C}$, muestran una moderada correlación con la variaciones del CP-2 ( $\mathrm{r}=0.59$ y r $=-0.53$, respectivamente).

\section{RESUMEN GENERAL, CONCLUSIONES Y PERSPECTIVAS}

El presente trabajo complementa los trabajos desarrollados por Tapley y Waylen (1990), Rome-Gaspaldy y Ronchail (1998) y Lagos et al. (2008), utilizando en total 155 estaciones de lluvia distribuidas en las tres vertientes del Perú: 85 en la vertiente del Pacífico (VP), 21 en la vertiente del Lago Titicaca (VT) y 49 en la vertiente del Amazonas (VA) para el periodo comprendido entre 1965-2007. Adicionalmente, nuestro trabajo contribuye en el análisis de la variabilidad de la TSM en diferentes regiones del Pacífico utilizando los índices C y E, recientemente definidos por Takahashi et al (2011).

Este estudio con un enfoque puramente estadístico ha llevado a sintetizar algunos alcances:

- Con la excepción de los dos eventos extremos (El Niño fuerte, ENF y La Niña fuerte, LNF), en dos de las tres vertientes (VP y VT) y para cierto número de estaciones localizadas en estas vertientes, no se encontraron suficiente número de estaciones con anomalías significativas para concluir que el IOS y su clasificación descrita en este artículo describa el patrón general del comportamiento de las lluvias en Perú. Además por la gran variabilidad de las lluvias en el Perú tanto en espacio como en tiempo (ver Espinoza et al., 2009 y Lavado et al. 2012a) los impactos del IOS son distintos y en la mayoría de los casos no sobrepasa el 50\% del total de estaciones en cada una de las tres vertientes analizadas.

- Los impactos de ENF describen fuertes anomalías positivas de precipitación en la zona norte de la VP así como anomalías negativas en el sur de la VP y la VT, ya reportados en la literatura científica (Waylen y Caviedes, 1986, Wells, 1987, Tapley y Waylen, 1990, Rome-Gaspaldy y Ronchail, 1998, Waylen y Poveda, 2002, entre otros). Sin embargo, en este estudio se describe que estas anomalías solo son observadas en $53 \%$ del total de estaciones en la VP y $52 \%$ en la VT, es decir, solo en la mitad del total de estaciones analizadas en estas vertientes se presentan anomalías significativas. Para los eventos LNF se presentan menor número de estaciones impactadas en la VP y VA (menos de $30 \%$ ) y es insignificante en la VT. Pero cabe destacar que contrariamente a lo que sucede con el ENF que produce anomalías positivas y negativas, los eventos $\mathrm{LN}$ provocan sobre todo anomalías positivas de precipitación en la mayoría de los casos.

- Del análisis estacional realizado en este trabajo podemos resaltar que los trimestres que presentaron estaciones con anomalías significativas en mayor número fueron DEF y MAM (estaciones lluviosas) ambos también para los eventos ENF y LNF pero sobre todo en la VP y VT. Destacándose los trimestres MAM, donde para los eventos ENF las anomalías llegaron hasta en +471 $\mathrm{mm}$ (zona norte de la VP) y el trimestre DEF para los eventos ENF en la VT donde las anomalías llegaron hasta en $-118 \mathrm{~mm}$.

Adicionalmente, en este trabajo se identifican los principales modos de variabilidad pluviométrica en el Perú mediante la técnica de funciones ortogonales empíricas. Estos principales modos son correlacionados tanto con el IOS y con los índices E y C. Los resultados muestran que el principal modo de variabilidad de las lluvias ( $37 \%$ de la varianza), caracterizado por fuertes lluvias en la costa peruana durante los eventos El Niño de 1983 y 1998, está altamente correlacionado con el índice $\mathrm{E}(\mathrm{r}=0.86)$, mientras que el segundo modo de variabilidad ( $25 \%$ de la varianza), caracterizado por mayores lluvias en la región andina, en la VT y VA durante los episodios La Niña, está moderadamente correlacionado con el IOS $(\mathrm{r}=0.59)$ y con el índice $\mathrm{C}(\mathrm{r}=-0.53)$.

En conclusión, la señal del IOS, no es relevante en caracterizar las lluvias, excepto en algunas estaciones y para algunas zonas características como el norte y sur de la VP y la VT. Los índices E y C resultan atractivos para la sintetización de la variabilidad de la TSM en el Pacífico y el índice E está 
bien correlacionado con el principal modo de variabilidad de las lluvias en el Perú. Las correlaciones entre la variabilidad de las lluvias en la región Andina, la VT y la VA, con el IOS y el índice $\mathrm{C}$, no alcanzan valores muy altos, lo cual sugiere que existen otros factores climáticos (no necesariamente en el Pacífico) que podrían explicar la variabilidad pluviométrica en estas regiones.

Recientemente, diversos estudios de las lluvias sobre el Perú muestran que existen otros componentes regionales que presentan teleconexiones significativas con las lluvias en el Perú como por ejemplo la TSM en el Atlántico tropical (Espinoza et al., 2009, Espinoza et al., 2011 y Lavado et al., 2012a) y que en conjunto con la circulación atmosférica han descrito climáticamente los extremos de lluvias en la cuenca Amazónica peruana. Así, teniendo como referencia los estudios previos, el presente trabajo coloca como perspectiva tratar de manera conjunta la variabilidad de la TSM en el Pacífico ecuatorial y en el Atlántico tropical, sugiriendo el análisis de la circulación atmosférica y sus mecanismos relacionados con las lluvias en el Perú.

\section{AGRADECIMIENTOS}

Esta investigación se realizó gracias a la iniciativa del Servicio Nacional de Meteorología e Hidrología del Perú, SENAMHI (www.senamhi.gob.pe) dentro de sus estudios "Impactos hidrológicos de los eventos El Niño y La Niña en Perú". Los autores agradecen a Ken Takahashi por la disponibilidad de los índices $\mathrm{E}$ y $\mathrm{C}$ usados en este estudio y a los dos revisores quienes han contribuido de manera sustancial en el resultado final del presente artículo.

\section{BIBLIOGRAFIA}

ACEITUNO, P.. On the functionning of the Southern Oscillation in the South American sector. Part 1 : surface climate. Monthly Weather Review, v. 116, p. 505-524, 1988.

ASHOK, K.; BEHERA, S. K.; RAO, S. A.; WENG, H.; YAMAGATA, T. El Niño Modoki and its possible teleconnection, Journal of Geophysical Research, v. 112, n. C11007, 2007. doi:10.1029/2006JC003798.

BJERKNES, J. A possible response of the atmospheric Hadley circulation to equatorial anomalies of ocean temperature. Tellus Series a-Dynamic Meteorology and Oceanography, v. 18, p. 820-829, 1966.

BJERKNES, J. Atmospheric teleconnections from the equatorial Pacific. Monthly Weather Review, v. 97, p. 163-172, 1969.

CARRILLO, C.. Hidrografía oceánica: Las corrientes oceánicas y estudios de la Corriente Peruana o de Humbolt. Boletin de la Sociedad Geográfica de Lima, v. 2, p. 72-110,1892.
CHIEW, F.; PIECHOTA, T.; DRACUP, J.; MCMAHON, T. El Niño/Southern Oscillation and Australian rainfall, streamflow and drought: Links and potential for forecasting. Journal of Hydrology, v. 204, p. 138-149, 1998.

CLUIS, D.; LABERGE, C. Analysis of the El Niño effect on the discharge of selected rivers in the Asia-Pacific region. Water International, v. 27, n. 2, p. 279-293, 2002.

DILLON, W.; GOLDSTEIN, M. Multivariate Analysis. Methods and applications. John Wiley and Sons editors, p. 157-186, 1984.

ESPINOZA, JC.; RONCHAIL, J.; FRAPPART, F.; LAVADO, W.; SANTINI, W.; GUYOT, JL. The major floods in the Amazonas River and tributaries (Western Amazon basin) during the 1970 - 2012 period: A focus on the 2012 flood. Journal of Hydrometeorology, 2013. doi: 10.1175/JHMD-12-0100.1.

ESPINOZA, J.C.; RONCHAIL, J.; GUYOT, J.L.; FILIZOLA, N.; NORIEGA, L.; LAVADO, C.W.S.; POMBOSA, R.; ROMERO, R. Spatio - Temporal rainfall variability in the Amazon Basin Countries (Brazil, Peru, Bolivia, Colombia and Ecuador). International Journal of Climatology, v. 29, p. 1574-1594, 2009.

ESPINOZA, J.C.; RONCHAIL, J.; GUYOT, J.L.; JUNQUAS, C.; VAUCHEL, P.; LAVADO, C.W.S.; DRAPEAU, G.; POMBOSA, R. Climate variability and extremes drought in the upper Solimões River (Western Amazon Basin): Understanding the exceptional 2010 drought. Geophysical Research Letters, v. 38, n. L13406, 2011. doi:10.1029/2011GL047862.

ESPINOZA, J.C.; RONCHAIL, J.; GUYOT, J.L.; JUNQUAS, C.; VAUCHEL, P.; LAVADO, C.W.S.; DRAPEAU, G.; POMBOSA, R. (2012). From drought to flooding: understanding the abrupt 2010-2011 hydrological annual cycle in the upper Solimões River (Western Amazon basin). Environmental Research Letters, v. 7, n. 2, 024008, 2012. doi:10.1088/1748-9326/7/2/024008.

KAWAMURA, A.; MCKERCHAR, A.; SPIGEL, R.; JINNO, K. 1998. Chaotic characteristics of the Southern Oscillation Index time series. Journal of Hydrology, v. 204, p. 168181, 1998.

LAGOS, P.; SILVA, Y.; NICKL, E.; MOSQUERA, K. El Niño-related precipitation variability in Perú. Advances in Geosciences, v. 14, p. 231-237, 2008.

LAVADO, C.W.S.; RONCHAIL, J.; LABAT, D.; ESPINOZA, J.C.; GUYOT, J.L. A basin-scale analysis of rainfall and runoff in Peru (1969-2004) Pacific, Titicaca and Amazonas drainages. Hydrological Sciences Journal, v. 57, n. 4, p. 1-18, 2012. doi:10.1080/02626667.2012.672985.

MARENGO, JA.; SOARES, W.; SAULO, C.; NICOLINI, M. Climatology of the low-level jet east of the Andes as 
derived from the NCEP-NCAR reanalyses: Characteristics and temporal variability. Journal of Climate, v. 17, p. 2261-2280, 2004.

MCKERCHAR, A.; PEARSON, C.; FITZHARRIS, B. Dependency of summer lake inflows and precipitation on spring SOI. Journal of Hydrology, v. 205, p. 66-80, 1998.

OPS. Perú: Fenómeno El Niño, 1997-1998. Organización Panamericana de la Salud. Fenómeno El Niño, 1997-1998, Washigton D.C., 2000

ROME-GASPALDY, S.; RONCHAIL, J. La pluviométrie au Pérou pendant les phases ENSO et LNSO. Bulletin de l'Institut Française d'Etudes Andins, v. 27, p. 675 - 685, 1998.

ROMERO, C.C.; BAIGORRIA, G.A.; STROOSNIJDER, L. Changes of erosive rainfall for El Nino and La Nina years in the northern Andean highlands of Peru. Climatic Change, v. 85, n. (3-4), p. 343-356, 2007.

ROPELEWSKI, C.F.; HALPERT, M.S. Global and Regional Scale Precipitation Patterns Associated with the El-Nino Southern Oscillation. Monthly Weather Review, v. 115, n. 8, p. 1606-1626, 1987.

SMITH, T.M.; REYNOLDS, R.W.; PETERSON, TC.; LAWRIMORE, J. Improvements to NOAA"s Historical Merged Land-Ocean Surface Temperature Analysis (18802006). Journal of Climate, v. 21, p. 2283-2296, 2008. DOI: 10.1175/2007JCLI2100.1

TAKAHASHI, K. The atmospheric circulation associated with extreme rainfall events in Piura, Peru, during the 1997 1998 and 2002 El Niño, Annales Geophysicae, v. 22, p. 3917-3926, 2004.

TAKAHASHI, K.; MONTECINOS, A.; GOUBANOVA, K.; DEWITTE, B. ENSO regimes: Reinterpreting the canonical and Modoki El Niño, Geophysical Research Letters, v. 38, L10704, 2011. doi:10.1029/2011GL047364.
TAPLEY, T.D.; WAYLEN, P.R. Spatial variability of Annual Precipitation and ENSO events in Western Peru. Hydrological Sciences Journal-Journal des Sciences Hydrologiques, v. 35, n. 4, p. 429-446, 1990.

VUILLE, M.; BRADLEY, R.S.; KEIMIG, F. Interannual climate variability in the Central Andes and its relation to tropical Pacific and Atlantic forcing. Journal of Geophysical Research-Atmospheres, v.105, n. D10, p. 12447-12460, 2000.

WANG, C.; DESER, C.; YU, J.-Y.; DINEZIO, P.; CLEMENT, A. El Niño-Southern Oscillation (ENSO): A review. In "Coral Reefs of the Eastern Pacific", P. Glymn, D. Manzello, and I. Enochs, Eds., Springer Science Publisher., 2012

WAYLEN, P.; POVEDA, G. El Nino-southern oscillation and aspects of western South American hydro-climatology. Hydrological Processes, v. 16, n. 6, p. 1247-1260, 2002.

WAYLEN, P.R.; CAVIEDES, C.N.. El Niño and annual floods on the north Peruvian littoral. Journal of Hydrology, v. 89, n. 1-2, p. 141-156, 1986.

WELLS, L.E.. An alluvial record of El-Niño events from northern coastal Peru. Journal of Geophysical ResearchOceans, v. 92, n. C13, p. 14463-14470, 1987.

XU, Z.X.; TAKEUCHI, K.; ISHIDAIRA, H. Correlation between El Nino-Southern Oscillation (ENSO) and precipitation in South-east Asia and the Pacific region. Hydrological Processes, v. 18, n. 1, p. 107-123, 2004.

YEH, S.W.; KUG, J.S.; DEWITTE, B.; KWON, M.H.; KIRTMAN, B. P.; JIN, F.F. El Niño in a changing climate, Nature, v. 461, p. 511-515, 2009. 